\title{
Fuzzy Inference Systems for Automatic Classification of Earthquake Damages
}

\author{
Petros-Fotios Alvanitopoulos ${ }^{1}$, Ioannis Andreadis ${ }^{1}$, and Anaxagoras Elenas ${ }^{2}$ \\ ${ }^{1}$ Laboratory of Electronics, Department of Electrical and Computer Engineering, \\ School of Engineering, Democritus University of Thrace, Xanthi, Greece \\ ${ }^{2}$ Institute of Structural Mechanics and Earthquake Engineering, Department of \\ Civil Enginnering, School of Engineering, Democritus University of Thrace, Xanthi, Greece \\ \{palvanit, iandread\}@ee.duth.gr, elenas@civil.duth.gr
}

\begin{abstract}
This paper presents efficient models in the area of damage potential classification of seismic signals. After an earthquake, one of the most important actions that authorities must take is to inspect structures and estimate the degree of damages. The interest is obvious for several reasons such as public safety, economical recourses management and infrastructure. This approach provides a comparative study between the Mamdani-type and Sugeno-type fuzzy inference systems (FIS). The fuzzy models use a set of artificial accelerograms in order to classify structural damages in a specific structure. Previous studies propose a set of twenty well-known seismic parameters which are essential for description of the seismic excitation. The proposed fuzzy systems use an input vector of twenty seismic parameters instead of the earthquake accelerogram and produce classification rates up to $90 \%$. Experimental results indicate that these systems are able to classify the structural damages in structures accurately. Both of them produce the same level of correct classification rates but the Mamdani-type has a slight superiority.
\end{abstract}

Keywords: Seismic Parameters, Earthquake Damage Classification, Fuzzy Inference Systems.

\section{Introduction}

Earthquake engineering can be defined as the field of engineering which deals with earthquakes and their effect on structures such as multistory buildings, bridges, towers, etc. As with any other natural phenomenon, earthquakes and their effects should be considered when designing a structure to ensure its safety. However, it is not economically feasible to design a structure that can withstand any seismic event without damages (elastic response). Sometimes devastating earthquakes hit populated areas. In this case, the ability to estimate the post-seismic damage status of oscillated structures instantly after the earthquake event may optimize the administration measures (optimized management of human and material resources, reduction of loss of lives and injuries).

The thrust of the current research is the automatic approach of the post-seismic status of buildings using intelligent FIS. Two types of fuzzy systems (Mamdani and Sugeno) 
are designed and implemented. These models use a set of artificial accelerograms. Numerical results indicate the ability of them to classify the structural damage in the examined structure.

In this research the set of artificial accelerograms is applied as a case study to a sixth floor reinforced concrete frame structure. The proposed classifiers can estimate the degree of structural damage on any other structure under the precondition that the training phase is carried out for the same building.

The key point of this study is to map the input space (artificial accelerograms) to an output space (degree of structural damage). Accelerograms are records of the acceleration versus time measured during an earthquake ground motion. The seismic accelerograms are a useful tool in earthquake engineering because they are able to provide an explicit description of the seismic excitation. However, due to the random sizes and shapes it is very difficult to exploit their similarities. Therefore, a set of twenty seismic parameters has been used to represent the seismic signals. These seismic parameters have been presented in the literature during the last decades and are able to express the damage potential of earthquakes. Table 1 presents those parameters. Previous study [1] reveals the correlation grade between seismic parameters and damage indices of structures.

Table 1. Seismic parameters

\begin{tabular}{ll}
\hline & Seismic Parameters \\
\hline Peak Ground Acceleration & Power $\left(\mathrm{P}_{0.90}\right)$ \\
Peak Ground Velocity & Central Period \\
Strong Motion Duration after Trifunac/Brady & Arias Intensity \\
Root Mean Square Acceleration & Spectral Displacement \\
Spectrum Intensity after Housner & Spectral Velocity \\
Spectrum Intensity after Kappos & Spectral Acceleration \\
Spectrum Intensity after Martinez-Rueda & Seismic Energy Input \\
Seismic Intensity after Fajfar/Vidic/Fischinger & Effective Peak Acceleration (EPA) \\
Destructiveness Potential & Maximum EPA \\
Cumulative Absolute Velocity & PGA to PGV ratio \\
\hline
\end{tabular}

For the present approach the level of structural damage caused by an earthquake is numerically quantified using damage indices (DIs). In recent years considerable efforts have been devoted to develop different types of damage indices (local-global). In general, a damage index is local when it refers to a restricted area (e.g. a cross section), while it is considered global when it describes the state of the whole structure. In this study the systems were developed based on the maximum inter storey drift ratio (MISDR) [2] and the widely used Park/Ang [3] overall damage indicator.

In order to quantify the consequences of an earthquake, it is necessary to know the physical deterioration (damage) of the building suffered during the seismic event. The examined set of 450 artificial accelerograms generates a wide spectrum of structural damage from negligible to severe. The values of damage indices of each accelerogram are divided into four damage categories (low-medium-large-total) according to Table 2. These classes correspond to non damage or minor damage, repairable damage, irreparable damage and partial or total collapse of the building, respectively. According to this classification of the structural damages it seems at first sight that the classification is 
Table 2. Damage Degree

\begin{tabular}{|c|c|c|c|c|}
\hline \multirow{2}{*}{$\begin{array}{c}\text { Structural Damage } \\
\text { Indicators }\end{array}$} & \multicolumn{4}{|c|}{ Damage Degree } \\
\hline & Low & Medium & Large & Total \\
\hline Park/Ang DI $_{G}[-]$ & $\leq 0.3$ & $0.3<\mathrm{DI}_{\mathrm{G}} \leq 0.6$ & $0.6<\mathrm{DI}_{\mathrm{G}} \leq 0.8$ & $\mathrm{DI}_{\mathrm{G}}>0.8$ \\
\hline MISDR [\%] & $\leq 0.5$ & $0.5<\operatorname{MISDR} \leq 1.5$ & $1.5<\operatorname{MISDR} \leq 2.5$ & $>2.5$ \\
\hline
\end{tabular}

unequivocal. However, from the engineer's point of view, a damage index value close to the class limits cannot be classified unequivocally to a specific damage category. For example, a structural damage ratio of 0.499 cannot unequivocally classify the accelerogram in the low class, because the option to belong in the medium class should be possible. Thus, the set of damage indices can be considered as a fuzzy set and fuzzy methods are appropriate to be applied to such classification procedures.

Previous studies [4-6] attempt to classify the structural damages in buildings. The first approach is based on the shape similarity of accelerograms. However, due to the random nature of seismic signals this approach led to a poor classification rates. The second approach is based on seismic parameters which can express the damage potential of an earthquake [1]. These seismic parameters were graphically used to represent the seismic load instead of the accelerogram itself. The extracted classification results based on artificial neural networks (ANNs) were better than the results of the first approach. Nevertheless, it was desirable to test the accuracy of the system using an additional damage index and a wide set of seismic signals. This has been achieved by the third technique using a combination of genetic algorithm and ANNs.

This paper studies the potential of two alternate soft computing techniques for earthquake damage classification based on fuzzy logic. Fuzzy classifiers have been successfully and widely applied to many fields. Moreover, recently the evolving fuzzy rule based classifiers have been proved promising tools that offer high performance (classification rates) and computational efficiency [7, 8]. The ability of fuzzy logic to model nonlinear functions of arbitrary complexity makes it even more important to investigate its ability to estimate the earthquake damage in buildings. The classification results show that these systems are able to estimate the seismic vulnerability of buildings avoiding complex mathematical processes.

\section{Proposed Method}

\subsection{The Mamdani-Type Fuzzy Inference System}

The proposed fuzzy system has twenty inputs corresponding to the twenty seismic parameters that result from the processing of seismic signals. These values can represent seismic accelerograms and are normalized into a range $[0,1]$. The FIS has one output that indicates the level of earthquake damage in structure. A model of the system is shown in Fig. 1.

The fuzzyfication of the inputs is realized using four Gaussian-shaped membership functions (mfs), namely low, medium, large and total. On the other hand the output consists of four triangular-shaped mfs, namely low, medium, large and total. The 
number and shape of the mfs were selected after an extensive set of preliminary tests. These tests involved simulations with different types and numbers of mfs such as triangular, trapezoidal and Gaussian. The tests confirmed that the below mfs presents good qualitative results. The following equations define the membership functions.

The Gausian function depends on two parameters $\sigma$ and $\mathrm{c}$ as given by:

$$
f(x, \sigma, c)=e^{\frac{-(x-c)^{2}}{2 \sigma^{2}}}
$$

where $\mathrm{c}$ and $\sigma$ are the mean value and the variance, respectively.

Low: $\mathrm{mf}_{1}(\mathrm{x} ; 0.1416,0)=\mathrm{e}^{\frac{-(\mathrm{x}-0)^{2}}{2 *(0.1416)^{2}}}$

Medium: $\mathrm{mf}_{2}(\mathrm{x} ; 0.1416,0.333)=\mathrm{e}^{\frac{-(\mathrm{x}-0.333)^{2}}{2 *(0.1416)^{2}}}$

Large: $\mathrm{mf}_{3}(\mathrm{x} ; 0.1416,0.667)=\mathrm{e}^{\frac{-(\mathrm{x}-0.667)^{2}}{2^{*}(0.1416)^{2}}}$

Total: $\mathrm{mf}_{4}(\mathrm{x} ; 0.1416,1)=\mathrm{e}^{\frac{-(\mathrm{x}-1)^{2}}{2 *(0.1416)^{2}}}$

The triangular function depends on two parameters $m$ and $\sigma$ as given by:

$$
f(x ; m, \sigma)=\left\{\begin{array}{lc}
1-\frac{m-x}{\sigma} & \text { for } m-\sigma \leq x \leq m \\
1-\frac{x-m}{\sigma} & \text { for } m \leq x \leq \sigma+m \\
0 & \text { otherwise }
\end{array}\right\}
$$

where $m$ and $\sigma$ are the center and width of the triangular-shaped function, respectively.

Low: $\operatorname{mf}_{1}(x ; 0,0.3333)=\left\{\begin{array}{ll}1-\frac{0-x}{0.3333} & \text { for }-0.3333 \leq x \leq 0 \\ 1-\frac{x-0}{0.3333} & \text { for } 0 \leq x \leq 0.3333 \\ 0 & \text { otherwise }\end{array}\right\}$

Medium: $\operatorname{mf}_{2}(x ; 0.3333,0.3333)=\left\{\begin{array}{ll}1-\frac{0.3333-x}{0.3333} & \text { for } 0 \leq x \leq 0.3333 \\ 1-\frac{x-0.3333}{0.3333} & \text { for } 0.3333 \leq x \leq 0.6667 \\ 0 \quad \text { otherwise }\end{array}\right\}$

Large: $\operatorname{mf}_{3}(\mathrm{x} ; 0.6667,0.3333)=\left\{\begin{array}{ll}1-\frac{0.6667-\mathrm{x}}{0.3333} & \text { for } 0.3333 \leq \mathrm{x} \leq 0.6667 \\ 1-\frac{\mathrm{x}-0.6667}{0.3333} & \text { for } 0.6667 \leq \mathrm{x} \leq 1 \\ 0 \quad \text { otherwise }\end{array}\right\}$ 
Total: $\operatorname{mf}_{4}(x ; 1,0.3333)=\left\{\begin{array}{ll}1-\frac{1-x}{0.3333} & \text { for } 0.6667 \leq x \leq 1 \\ 1-\frac{x-1}{0.3333} & \text { for } 1 \leq x \leq 1.3333 \\ 0 & \text { otherwise }\end{array}\right\}$

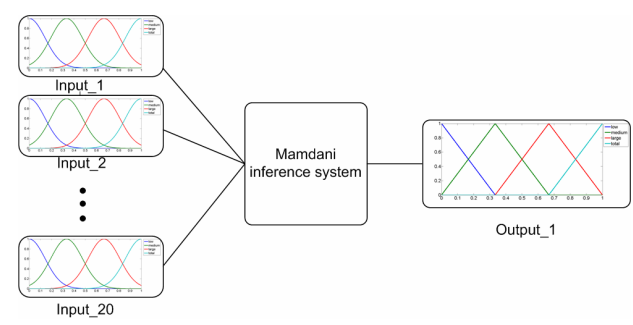

Fig. 1. The Mamdani inference system

The inputs are routed to the output through a Mamdani [9] type of fuzzy inference that uses a set of rules which is not unique and depends on the number of the training samples. In this study two DIs have been used so the structure of the model has a different set of rules for each damage indicator. Knowing the training data points it is possible to extract fuzzy rules [10]. In an effort to generate fuzzy rules the following steps have been considered:

Step 1: Divide the input/output space into fuzzy regions and assign each region a fuzzy membership function. In this approach the number of regions for each variable is equal to four since four are the aforementioned membership functions.

Step 2: Generate fuzzy rules using the training samples. First determine the degree of the given training input $\mathrm{X}$ for each membership function, and assign the input to the region with the maximum degree of membership. The extracted rule is as follows:

$\mathrm{R}_{1}$ : If $\mathrm{X}_{1}$ is Low ${ }^{\wedge} \mathrm{X}_{2}$ is Large . . $\mathrm{X}_{20}$ is Medium, THEN $\mathrm{Y}$ is Low

Step 3: Assign a degree to each rule: For each data point a fuzzy rule is generated. The degree of the fuzzy rule is:

$$
\mathrm{D}\left(\mathrm{R}_{1}\right)=\min \left(\mu \operatorname{Low}\left(\mathrm{X}_{1}\right), \mu \operatorname{Large}\left(\mathrm{X}_{2}\right), \ldots \mu \operatorname{Medium}\left(\mathrm{X}_{20}\right)\right)
$$

In this model the antecedent of each rule has more than one part. The antecedent consists of twenty terms since twenty are the seismic parameters. The fuzzy operator AND (min) is applied to obtain a number that represents the result of the antecedent of each rule. In Mamdani type of fuzzy inference, the fuzzy sets from the consequent of each rule are combined through the aggregation operator and the resulting fuzzy set is defuzzified to yield the output of the system. The aggregation operator, in this case, is set to maximum and the defuzzification method is that of the center of gravity (CoG) [10].

\subsection{The Tagaki-Sugeno-Kang (TSK)-Type Fuzzy Inference System}

In the present study a (TSK) fuzzy system was used for the classification of earthquake damage in the examined structure. This methodology was introduced in 1985 
[11], and it is similar to the Mamdani method in many respects. The first two parts of the fuzzy inference process, fuzzifying the inputs and applying the fuzzy operator, are exactly the same. The main difference between Mamdani and Sugeno is that the Sugeno output membership functions are either linear or constant.

Let $D$ be the set of input-output data pairs (training data), $X=\left\{x_{1}, \ldots, x_{n}\right\} \subset \mathbb{R}^{\mathrm{p}}$ be the set of $p$-dimensional input vectors, and $Y=\left\{y_{1}, \ldots, y_{n}\right\} \subset \mathbb{R}$ be the associated set of output vectors. The set $Z$ with input-output vectors taken together can be denoted by:

$$
\mathrm{Z}=\left\{\mathrm{z}_{\mathrm{k}}=\left(\begin{array}{c}
\mathrm{x}_{\mathrm{k}} \in \mathfrak{R}^{\mathrm{p}} \\
\mathrm{y}_{\mathrm{k}} \in \mathfrak{R}
\end{array}\right) \in \mathfrak{R}^{\mathrm{p}+1}, \mathrm{k}=1, \ldots, \mathrm{n}\right\}
$$

The TSK model comprises of a set of IF-THEN fuzzy rules having the following form:

$\mathrm{R}_{1}$ : If $x_{1}$ is $A_{i l}$ and $\ldots$ and $x_{p}$ is $A_{i p}$ Then $y_{i}=b_{i 0}+b_{i 1} x_{1}+\ldots+b_{i p} x_{p}$ where $i=1, \ldots, M, A_{i j}=(j=1, \ldots, p)$ are antecedent fuzzy sets, $y_{i}$ is the output of the $i$ th rule, and $b_{i l}(l=0,1, \ldots, p)$ are the consequent parameters. The overall output $y^{\text {overall }}$ of the model is computed as follows:

$$
\mathrm{y}^{\text {overall }}=\frac{\sum_{\mathrm{i}=1}^{\mathrm{M}} \tau_{\mathrm{i}} \mathrm{y}_{\mathrm{i}}}{\sum_{\mathrm{i}=1}^{\mathrm{M}} \tau_{\mathrm{i}}}=\frac{\sum_{\mathrm{i}=1}^{\mathrm{M}} \tau_{\mathrm{i}}\left(\mathrm{b}_{\mathrm{i} 0}+\mathrm{b}_{\mathrm{i} 1} \mathrm{x}_{1}+\ldots+\mathrm{b}_{\mathrm{ip}} \mathrm{x}_{\mathrm{p}}\right)}{\sum_{\mathrm{i}=1}^{\mathrm{M}} \tau_{\mathrm{i}}}
$$

where $\tau_{i}$ is the firing strength of $R_{i}$.

In our approach the proposed model is a zero-order TSK system. The consequent parameters are set to 0 (except $b_{i 0}$ ) and the outputs of rules are constant. The rule base is the same with the Mamdani fuzzy model. The mfs of the input vector are set using the previous equations (2-5). All consequent mfs from Mamdani are replaced by singleton spikes. The firing strength of each rule is computed using the AND operator, in this case, is set to minimum. The TSK model is shown in Fig. 2.

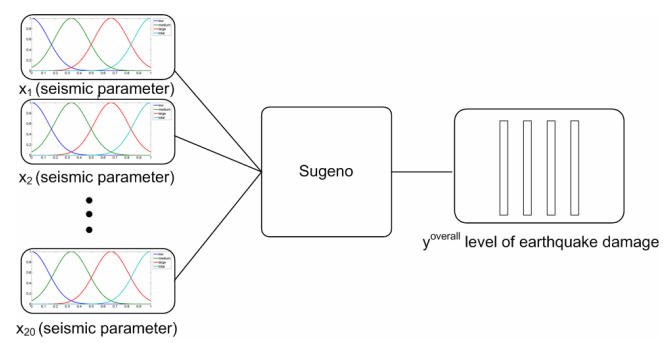

Fig. 2. The TSK fuzzy system

\section{Results}

After the nonlinear dynamic analysis of the structure, two DIs, namely, the DI of Park/Ang, and the MISDR have been computed. According to these DIs, the damages caused by seismic excitations, were classified into four classes. The next step of 
our method was to pass the seismic signals through the proposed fuzzy models and examine their effectiveness. Artificial accelerograms were used in order to simulate natural earthquakes. Table 3 presents the classification results for the examined FIS. As it can be seen, from Table 3 the same set of training samples provide different number of rules for each DI. The correct classification rates were better in the case of Park/Ang DI than in the case of MISDR. Moreover, the Mamdami system presents better classification rates than the TSK model. However, both of these systems are able to evaluate the seismic damage potential and produce the same level of correct classification rates.

Table 3. Classification Results Using Mamdani and TSK Fuzzy Systems

\begin{tabular}{lcccc}
\hline \multicolumn{1}{c}{ Type of FIS } & \multicolumn{2}{c}{ Mamdani } & \multicolumn{2}{c}{ TSK } \\
\hline & MISDR & DI of Park/Ang & MISDR & DI of Park/Ang \\
\cline { 2 - 5 } Number of training samples & 300 & 300 & 300 & 300 \\
Number of Rules & 193 & 179 & 193 & 179 \\
Number of unknown samples & 450 & 450 & 450 & 450 \\
Number of well recognized samples & 392 & 407 & 388 & 393 \\
Total \% of the classification & $87.10 \%$ & $90,44 \%$ & $86,22 \%$ & $87,33 \%$ \\
\hline
\end{tabular}

\section{Conclusions}

The utility of fuzzy logic in pattern classification problems is nowadays quite extensive. This paper attempts to design diagnostic systems with high classification accuracy in the scientific area of earthquake engineering. First a set of 450 artificial accelerograms with known damage effects was required in order to derive the parameters which are able to describe the seismic intensity. Two FIS (Mamdani-type, TSK-type) are presented. The Mamdani-type is slightly better. Experimental results show that the systems developed herein, presents the same level of classification rates (up to 90\%). These promising results are the starting point for further exploration with more seismic events applied also to other types of structures. Until today, survey is performed with on-site examination by expert engineers. With the proposed technique engineers will have an additional tool which can guide them to a fast and confident estimation of the post-seismic damage condition of interested structures. Moreover, they can be used by the public administration for the adequate post-seismic distribution of financial and other resources in the case of severe earthquakes.

\section{References}

1. Elenas, A., Meskouris, K.: Correlation Study Between Seismic Acceleration Parameters and Damage Indices of Structures. J. Engineering Structures 23, 698-704 (2001)

2. Rodriguez-Gomez, S., Cakmak, A.S.: Evaluation of Seismic Damage Indices for Reinforced Concrete Structures. Technical Report NCEER-90-0022, State University of New York, Buffalo (1990) 
3. Park, Y.J., Ang, A.H.S.: Mechanistic Seismic Damage Model for Reinforced Concrete. J. Structural Engineering 111, 722-739 (1985)

4. Andreadis, I., Tsiftzis, Y., Elenas, A.: Intelligent Seismic Acceleration Signal Processing for Damage Classification in Buildings. J. IEEE Transactions on Instrumentation and Measurement 56, 1555-1564 (2007)

5. Alvanitopoulos, P., Andreadis, I., Elenas, A.: A New Algorithm for the Classification of Earthquake Damages in Structures. In: 5th IASTED International Conference on Signal Processing, Pattern Recognition and Applications, Innsbruck, Austria, pp. 151-156 (February 2008)

6. Alvanitopoulos, P., Andreadis, I., Elenas, A.: A Genetic Algorithm for the Classification of Earthquake Damages in Buildings. In: 5th IFIP Conference on Artificial Intelligence Applications \& Innovations, Thessaloniki, Greece, pp. 341-346 (April 2009)

7. Angelov, P., Zhou, X.: Evolving Fuzzy-Rule-Based Classifiers from Data Streams. J. IEEE Transactions on Fuzzy Systems 16, 1462-1475 (2008)

8. Angelov, P., Lughofer, E., Zhou, X.: Evolving Fuzzy Classifiers Using Different Model Architectures. J. Fuzzy Sets and Systems 159, 3160-3182 (2008)

9. Mamdani, E.H., Assilian, S.: An Experiment in Linguistic Synthesis with a Fuzzy Logic Controller. J. Man-Machine Studies 7, 1-13 (1975)

10. Kulkarni, A.D.: Computer Vision and Fuzzy-Neural Systems. Prentice Hall PTR, Upper Saddle River (2001)

11. Sugeno, M.: Industrial Applications of Fuzzy Control. Elsevier Science, New York (1985) 